\title{
Table des matières
}

4

\section{Éditorial}

Karolina Kosińska

\section{L'ARCHITECTURE ET LA NARRATION}

L'architectonique du théâtre - la structure du film - les recoins de l'esprit. Les passages new-yorkais de Birdman Grzegorz Nadgrodkiewicz

L'architecture comme l'intrigue. Sur Parasite de Bong Joon-ho Rafał Koschany

Les corridors interminables. Les paradoxes d'architecture dans le film L'année dernière à Marienbad d'Alain Resnais

Paulina Kwiatkowska

Le musée en deuil. Les élégies architecturales d'Alexandre Sokourov Natasza Korczarowska

\section{LE CADRAGE DE LA SOCIÉTÉ}

Cadre de vie. Deux ou trois choses que je sais d'elle (1967) de Jean-Luc Godard, la télévision française et le discours architectural Jacqueline Maurer

L'architecture, l'urbanisme et la communauté - Byker de Sirkka-Liisa Konttinen Karolina Kosińska

L'architecture et le colonialisme japonais - l'image filmique de l'espace urbain à Séoul occupée Krzysztof Loska

Les banlieues américaines à l'ombre de la grande ville Patrycja Włodek

\section{LA CORRESPONDANCE DES MÉDIAS}

Les designers de l'espace-temps. L'architecture moderne dans le cadre filmique

\section{Katarzyna Uchowicz}

Construit comme pluie. L'analyse cinématographique des spéculations architecturales non constructibles - une étude de cas d'Instant City (réal. Peter Cook et Ron Herron, 1968) et de Le Théorème zéro (réal. Terry Gilliam, 2013)

Maciej Stasiowski

Les espaces semi-filmiques. La simulation des propriétés des espaces cinématographiques dans les jeux vidéo Joanna Sikorska

\section{PAR L'OEIL ET PAR L'OREILLE}

L'âge d'or - un chef-d'œuvre de provocation Marcin Giżycki

\section{LES LIVRES SUR LE CINÉMA}

Bolesławski, le vôtre et le nôtre Teresa Rutkowska

Comment les écrans sont-ils devenus des livres Andrzej Gwóźdź

Sur la subjectivisation dans un film encore une fois Tomasz Kłys

Les histoires des émigrants polonais dans le cinéma européen Krzysztof Loska

Table of contents

Table des matières 